\title{
Supporting Information \\ for Importance of Chiral Phase-Transfer Catalysts with Dual Functions in Obtaining High Enantioselectivity in the Michael Reaction of Malonates and Chalcone Derivatives
}

\author{
Takashi Ooi, Daisuke Ohara, Kazuhiro Fukumoto, and Keiji Maruoka* \\ Department of Chemistry, Graduate School of Science, Kyoto University \\ Sakyo, Kyoto 606-8502, Japan
}

Preparation and Characterization of Chiral Quaternary Ammonium Bromide 2. To a solution of 1 ( $50 \mathrm{mg}, 0.025 \mathrm{mmol})^{1}$ in $\mathrm{CH}_{2} \mathrm{Cl}_{2}(5.0 \mathrm{~mL})$ was added boron trifluoride-diethyl etherate $(16 \mu \mathrm{L}, 0.125 \mathrm{mmol})$ dropwise at $0{ }^{\circ} \mathrm{C}$ under argon atmosphere. After being stirred for $2 \mathrm{~h}$ at room temperature, triethylsilane $(40 \mu \mathrm{L}, 0.25 \mathrm{mmol})$ was introduced. The reaction mixture was then heated to reflux and stirred there for $24 \mathrm{~h}$. The resulting mixture was poured into water and extracted with $\mathrm{CH}_{2} \mathrm{Cl}_{2}$. The organic phase was dried over $\mathrm{Na}_{2} \mathrm{SO}_{4}$ and concentrated. The residual crude ammonium salt was passed through a column of ion exchange resin, Amberlyst A-26 $\left(\mathrm{OH}^{-}\right)$, with $\mathrm{MeOH}$ as eluant to convert it into the corresponding hydroxide, which was subsequently neutralized with 2 equiv of $1 \mathrm{~N} \mathrm{HBr}$ solution. After removal of solvents, the residue was purified by column chromatography on silica gel $\left(\mathrm{MeOH} / \mathrm{CH}_{2} \mathrm{Cl}_{2}=1: 10\right.$ as eluant) to give 2 (22.4 $\mathrm{mg}, 0.012$ mmol, $48 \%$ yield): $[\alpha]_{\mathrm{D}}{ }^{29}-7.92{ }^{\circ}\left(c 0.42, \mathrm{CHCl}_{3}\right) ;{ }^{1} \mathrm{H} \mathrm{NMR}\left(400 \mathrm{MHz}, \mathrm{CDCl}_{3}\right) \delta 7.93(2 \mathrm{H}, \mathrm{d}, J=$ 8.4 Hz, Ar-H), 7.89 (2H, s, Ar-H), 7.73-7.52 (18H, m, Ar-H), 7.48-7.33 (24H, m, Ar-H), 7.29-7.22 (20H, m, Ar-H), 7.18-7.16 (12H, m, Ar-H), 7.11-7.08 (12H, m, Ar-H), 6.92 (4H, s, Ar-H), 5.88 (2H, s, $\left.\mathrm{Ar}_{3} \mathrm{CH}\right), 4.77\left(2 \mathrm{H}, \mathrm{d}, J=13.6 \mathrm{~Hz}, \mathrm{CH}_{2}\right), 4.66\left(2 \mathrm{H}, \mathrm{d}, J=12.8 \mathrm{~Hz}, \mathrm{CH}_{2}\right), 4.51(2 \mathrm{H}, \mathrm{d}, J=13.6 \mathrm{~Hz}$, $\left.\mathrm{CH}_{2}\right), 4.08\left(2 \mathrm{H}, \mathrm{d}, J=12.8 \mathrm{~Hz}, \mathrm{CH}_{2}\right) ;{ }^{13} \mathrm{C} \mathrm{NMR}\left(100 \mathrm{MHz}, \mathrm{CDCl}_{3}\right) \delta 144.0,143.6,142.9,142.7$, $142.1,141.9,141.8,140.6,139.9,139.8,139.6$, 135.6, 132.2, 132.0, 131.9, 131.8, 131.2, 130.8, $129.0,128.6,128.5,128.4,128.2$, 127.7, 127.5, 127.3, 127.2, 126.9, 126.8, 126.6, 126.4, 126.2, 125.7, 125.6, 124.5, 124.3, 64.9, 60.3, 53.1, 29.6; IR (neat) 3057, 3034, 2924, 2853, 1593, 1576, 1497, 1454, 1429, 1029, 912, 883, 758, $696 \mathrm{~cm}^{-1}$; HRMS (ESI) Calcd for $\mathrm{C}_{146} \mathrm{H}_{104} \mathrm{~N}\left(\mathrm{M}^{+}\right)$: 1870.8163 , Found: $1870,8172$.

General Procedure for Enantioselective Michael Addition of Diethyl Malonate to Chalcone Derivative Using Designer Chiral Phase-Transfer Catalyst 1. To a mixture of 1,4-diarylenone $3(0.1 \mathrm{mmol}), \mathrm{K}_{2} \mathrm{CO}_{3}(1.6 \mathrm{mg}, 10 \mathrm{~mol} \%)$ and chiral quaternary ammonium salt $1(6 \mathrm{mg}, 3 \mathrm{~mol} \%)$ in toluene $(0.2 \mathrm{~mL})$ was added diethyl malonate $(72 \mu \mathrm{L}, 0.4 \mathrm{mmol})$ at $-20{ }^{\circ} \mathrm{C}$ under argon atmosphere. The reaction mixture was stirred for $24 \mathrm{~h}$ at that temperarure, and then water and ether were added sequentially. The ethereal phase was separated, washed with brine and dried over $\mathrm{Na}_{2} \mathrm{SO}_{4}$. Evaporation of solvents gave the crude product, which was purified by column chromatography on silica gel (ethyl acetate/hexane as eluant) to afford the corresponding Michael adduct 4 . An 
enantiomeric excess of $\mathbf{4}$ was determined by chiral stationary-phase HPLC analysis.

\section{Characterization of Michael Adduct 4.}

2-(1-Naphthalen-2-yl-3-oxo-3-phenylpropyl)malonic Acid Diethyl Ester $(4 \mathbf{b}):[\alpha]_{\mathrm{D}}{ }^{20}-8.37^{\circ}$ $\left[c\right.$ 0.87, $\mathrm{CHCl}_{3}\left(91 \%\right.$ ee)]; ${ }^{1} \mathrm{H} \mathrm{NMR}\left(400 \mathrm{MHz}, \mathrm{CDCl}_{3}\right) \delta 7.89(2 \mathrm{H}, \mathrm{d}, J=7.9 \mathrm{~Hz}, \mathrm{Ar}-\mathrm{H}), 7.75-7.70$ (4H, m, Ar-H), 7.49 (1H, t, J= 7.9 Hz, Ar-H), 7.45-7.36 (5H, m, Ar-H), 4.37 (1H, dt, J= 9.1, $5.1 \mathrm{~Hz}$, $\left.\mathrm{COCH}_{2} \mathrm{CH}\right), 4.20\left(2 \mathrm{H}, \mathrm{q}, J=7.1 \mathrm{~Hz}, \mathrm{CO}_{2} \mathrm{CH}_{2}\right), 3.94\left(1 \mathrm{H}, \mathrm{d}, J=9.9 \mathrm{~Hz}, \mathrm{CHCO}_{2}\right), 3.89(2 \mathrm{H}, \mathrm{q}, J=$ $\left.7.1 \mathrm{~Hz}, \mathrm{CO}_{2} \mathrm{CH}_{2}\right), 3.63\left(1 \mathrm{H}, \mathrm{dd}, J=16.6,5.1 \mathrm{~Hz}, \mathrm{COCH}_{2}\right), 3.58\left(1 \mathrm{H}, \mathrm{dd}, J=16.6,9.1 \mathrm{~Hz}, \mathrm{COCH}_{2}\right)$, $1.22\left(3 \mathrm{H}, \mathrm{t}, J=7.1 \mathrm{~Hz}, \mathrm{CH}_{3}\right), 0.92\left(3 \mathrm{H}, \mathrm{t}, J=7.1 \mathrm{~Hz}, \mathrm{CH}_{3}\right) ;{ }^{13} \mathrm{C} \mathrm{NMR}\left(100 \mathrm{MHz}, \mathrm{CDCl}_{3}\right) \delta 197.1$, 137.9, 136.6, 133.1, 132.8, 132.4, 128.4, 127.9, 127.9, 127.6, 127.4, 126.9, 126.2, 125.8, 125.5, 61.6, 61.3, 42.6, 40.8, 14.1, 13.8; IR (neat) 1728, 1684, 1597, 1506, 1449, 1368, 1256, 1219, 1152, 1096, 1028, 858, 820, 748, 691, $650 \mathrm{~cm}^{-1}$; HRMS (ESI) Calcd for $\mathrm{C}_{26} \mathrm{H}_{26} \mathrm{NaO}_{5}\left([\mathrm{M}+\mathrm{Na}]^{+}\right): 441.1672$, Found: 441.1673; HPLC analysis: DAICEL Chiralpak AD-H, hexane/ethanol $=4: 1$, flow rate $=1.0$ $\mathrm{mL} / \mathrm{min}$, retention time; $28.5 \mathrm{~min}$ (minor) and $31.4 \mathrm{~min}$ (major).

2-[1-(4-Methoxyphenyl)-3-oxo-3-phenylpropyl]malonic Acid Diethyl Ester (4c): $[\alpha]_{\mathrm{D}}{ }^{19}$ $-16.5^{\circ}\left[c\right.$ 3.40, $\mathrm{CHCl}_{3}$ (87\% ee)]; ${ }^{1} \mathrm{H} \mathrm{NMR}\left(400 \mathrm{MHz}, \mathrm{CDCl}_{3}\right) \delta 7.89(2 \mathrm{H}, \mathrm{d}, J=7.5 \mathrm{~Hz}, \mathrm{Ar}-\mathrm{H})$, $7.52(1 \mathrm{H}, \mathrm{t}, J=7.5 \mathrm{~Hz}, \mathrm{Ar}-\mathrm{H}), 7.41(2 \mathrm{H}, \mathrm{t}, J=7.5 \mathrm{~Hz}, \mathrm{Ar}-\mathrm{H}), 7.17(2 \mathrm{H}, \mathrm{t}, J=8.7 \mathrm{~Hz}, \mathrm{Ar}-\mathrm{H}), 6.76$ $(2 \mathrm{H}, \mathrm{t}, J=8.7 \mathrm{~Hz}, \mathrm{Ar}-\mathrm{H}), 4.24-4.15\left(2 \mathrm{H}, \mathrm{m}, \mathrm{CO}_{2} \mathrm{CH}_{2}\right), 4.13\left(1 \mathrm{H}, \mathrm{dt}, J=9.9,4.4 \mathrm{~Hz}, \mathrm{COCH}_{2} \mathrm{C} \underline{\mathrm{H}}\right)$, $3.96\left(2 \mathrm{H}, \mathrm{q}, J=7.1 \mathrm{~Hz}, \mathrm{CO}_{2} \mathrm{CH}_{2}\right), 3.78\left(1 \mathrm{H}, \mathrm{d}, J=9.9 \mathrm{~Hz}, \mathrm{CHCO}_{2}\right), 3.73\left(3 \mathrm{H}, \mathrm{s}, \mathrm{OCH}_{3}\right), 3.51(1 \mathrm{H}$, $\left.\mathrm{dd}, J=16.6,7.1 \mathrm{~Hz}, \mathrm{COCH}_{2}\right), 3.40\left(1 \mathrm{H}, \mathrm{dd}, J=16.6,9.9 \mathrm{~Hz}, \mathrm{COCH}_{2}\right), 1.24\left(3 \mathrm{H}, \mathrm{t}, J=7.1 \mathrm{~Hz}, \mathrm{CH}_{3}\right)$, $1.03\left(3 \mathrm{H}, \mathrm{t}, J=7.1 \mathrm{~Hz}, \mathrm{CH}_{3}\right) ;{ }^{13} \mathrm{C}$ NMR $\left(100 \mathrm{MHz}, \mathrm{CDCl}_{3}\right) \delta 197.4,168.2,167.5,158.3,136.7$, $132.8,132.2,129.1,128.4,127.9,113.6,61.6,61.3,57.8,55.1,42.8,40.2,14.1,13.9$; IR (neat) 1728, 1684, 1611, 1597, 1581, 1514, 1464, 1449, 1368, 1248, 1179, 1152, 1113, 1096, 1032, 916, 860, 831, 733, $691 \mathrm{~cm}^{-1}$; HRMS (ESI) Calcd for $\mathrm{C}_{23} \mathrm{H}_{26} \mathrm{NaO}_{6}\left([\mathrm{M}+\mathrm{Na}]^{+}\right)$: 421.1622, Found: 421.1621; HPLC analysis: DAICEL Chiralpak AD-H, hexane/ethanol $=20: 1$, flow rate $=1.0$ $\mathrm{mL} / \mathrm{min}$, retention time; $117 \mathrm{~min}$ (minor) and $155 \mathrm{~min}$ (major).

2-(1-[1,3]-Benzodioxol-5-yl-3-oxo-3-phenylpropyl)malonic Acid Diethyl Ester (4d): $[\alpha]_{\mathrm{D}}{ }^{21}$ $-14.1^{\circ}\left[c\right.$ 1.96, $\mathrm{CHCl}_{3}(89 \%$ ee $\left.)\right] ;{ }^{1} \mathrm{H} \mathrm{NMR}\left(400 \mathrm{MHz}, \mathrm{CDCl}_{3}\right) \delta 7.90(2 \mathrm{H}, \mathrm{d}, J=7.5 \mathrm{~Hz}, \mathrm{Ar}-\mathrm{H})$, $7.52(1 \mathrm{H}, \mathrm{t}, J=7.5 \mathrm{~Hz}, \operatorname{Ar}-\mathrm{H}), 7.42(2 \mathrm{H}, \mathrm{t}, J=7.5 \mathrm{~Hz}, \operatorname{Ar}-\mathrm{H}), 6.76(1 \mathrm{H}, \mathrm{s}, \operatorname{Ar}-\mathrm{H}), 6.71(1 \mathrm{H}, \mathrm{d}, J=$ $7.9 \mathrm{~Hz}, \mathrm{Ar}-\mathrm{H}), 6.65(1 \mathrm{H}, \mathrm{d}, J=7.9 \mathrm{~Hz}, \mathrm{Ar}-\mathrm{H}), 5.87\left(2 \mathrm{H}, \mathrm{s}, \mathrm{OCH}_{3}\right), 4.23-4.16\left(2 \mathrm{H}, \mathrm{m}, \mathrm{CO}_{2} \mathrm{CH}_{2}\right), 4.11$ $\left(1 \mathrm{H}, \mathrm{dt}, J=9.4,4.4 \mathrm{~Hz}, \mathrm{COCH}_{2} \mathrm{C} \underline{\mathrm{H}}\right), 4.00\left(2 \mathrm{H}, \mathrm{q}, J=7.1 \mathrm{~Hz}, \mathrm{CO}_{2} \mathrm{CH}_{2}\right), 3.75(1 \mathrm{H}, \mathrm{d}, J=9.4 \mathrm{~Hz}$, $\left.\mathrm{CHCO}_{2}\right), 3.50\left(1 \mathrm{H}, \mathrm{dd}, J=16.6,4.4 \mathrm{~Hz}, \mathrm{COCH}_{2}\right), 3.39\left(1 \mathrm{H}, \mathrm{dd}, J=16.6,9.4 \mathrm{~Hz}, \mathrm{COCH}_{2}\right), 1.25(3 \mathrm{H}$, $\left.\mathrm{t}, J=7.1 \mathrm{~Hz}, \mathrm{CH}_{3}\right), 1.07\left(3 \mathrm{H}, \mathrm{t}, J=7.1 \mathrm{~Hz}, \mathrm{CH}_{3}\right) ;{ }^{13} \mathrm{C} \mathrm{NMR}\left(100 \mathrm{MHz}, \mathrm{CDCl}_{3}\right) \delta 197.2,168.0$, 167.4, 147.3, 146.3, 136.6, 134.0, 132.9, 128.4, 127.9, 121.3, 108.5, 108.0, 100.8, 61.6, 61.3, 57.7, 42.7, 40.6, 14.1, 13.9; IR (neat) 1728, 1684, 1506, 1489, 1447, 1368, 1339, 1236, 1179, 1153, 1099, 1036, 934, 910, 858, 810, 729, $691 \mathrm{~cm}^{-1}$; HRMS (ESI) Calcd for $\mathrm{C}_{23} \mathrm{H}_{24} \mathrm{NaO}_{7}\left([\mathrm{M}+\mathrm{Na}]^{+}\right): 435.1414$, 
Found: 435.1412; HPLC analysis: DAICEL Chiralpak AD-H, hexane/ethanol $=4: 1$, flow rate $=1.0$ $\mathrm{mL} / \mathrm{min}$, retention time; $47.3 \mathrm{~min}$ (minor) and $53.5 \mathrm{~min}$ (major).

2-[1-(4-Chlorophenyl)-3-oxo-3-phenylpropyl]malonic Acid Diethyl Ester (4e): $[\alpha]_{\mathrm{D}}{ }^{22}-22.7^{\circ}$ [c 0.60, $\mathrm{CHCl}_{3}\left(85 \%\right.$ ee)]; ${ }^{1} \mathrm{H} \mathrm{NMR}\left(400 \mathrm{MHz}, \mathrm{CDCl}_{3}\right) \delta 7.88(2 \mathrm{H}, \mathrm{d}, J=7.5 \mathrm{~Hz}, \mathrm{Ar}-\mathrm{H}), 7.53(1 \mathrm{H}, \mathrm{t}$, $J=7.5 \mathrm{~Hz}, \mathrm{Ar}-\mathrm{H}), 7.42(2 \mathrm{H}, \mathrm{t}, J=7.5 \mathrm{~Hz}, \mathrm{Ar}-\mathrm{H}), 7.21(4 \mathrm{H}, \mathrm{s}, \mathrm{Ar}-\mathrm{H}), 4.24-4.13\left(3 \mathrm{H}, \mathrm{m}, \mathrm{COCH}_{2} \mathrm{C}\right.$ and $\left.\mathrm{CO}_{2} \mathrm{CH}_{2}\right), 3.98\left(2 \mathrm{H}, \mathrm{q}, J=7.1 \mathrm{~Hz}, \mathrm{CO}_{2} \mathrm{CH}_{2}\right), 3.78\left(1 \mathrm{H}, \mathrm{d}, J=9.8 \mathrm{~Hz}, \mathrm{CHCO}_{2}\right), 3.53(1 \mathrm{H}, \mathrm{dd}, J=$ 16.6, $\left.4.4 \mathrm{~Hz}, \mathrm{COCH}_{2}\right), 3.43\left(1 \mathrm{H}, \mathrm{dd}, J=16.6,9.8 \mathrm{~Hz}, \mathrm{COCH}_{2}\right), 1.24\left(3 \mathrm{H}, \mathrm{t}, J=7.1 \mathrm{~Hz}, \mathrm{CH}_{3}\right), 1.05$ $\left(3 \mathrm{H}, \mathrm{t}, J=7.1 \mathrm{~Hz}, \mathrm{CH}_{3}\right) ;{ }^{13} \mathrm{C} \mathrm{NMR}\left(100 \mathrm{MHz}, \mathrm{CDCl}_{3}\right) \delta 197.0,167.9$. 167.3, 138.9, 136.5, 133.0, 132.7, 129.6, 128.5, 128.4, 127.9, 61.7, 61.5, 57.3, 42.4, 40.2, 14.1, 13.9; IR (neat) 1728, 1686, 1597, 1580, 1491, 1449, 1414, 1368, 1252, 1227, 1153, 1094, 1030, 1015, 860, 829, 754, $691 \mathrm{~cm}^{-1}$; HRMS (ESI) Calcd for $\mathrm{C}_{22} \mathrm{H}_{23} \mathrm{ClNaO}_{5}\left([\mathrm{M}+\mathrm{Na}]^{+}\right)$: 425.1126, Found: 425.1128; HPLC analysis: DAICEL Chiralpak AD-H, hexane/ethanol $=20: 1$, flow rate $=1.0 \mathrm{~mL} / \mathrm{min}$, retention time; $65.5 \mathrm{~min}$ (minor) and $90.8 \mathrm{~min}$ (major).

2-[3-(4-Chlorophenyl)-3-oxo-1-phenylpropyl]malonic Acid Diethyl Ester (4f): $[\alpha]_{\mathrm{D}}{ }^{19}-17.2^{\circ}$ [c 3.70, $\mathrm{CHCl}_{3}\left(86 \%\right.$ ee)]; ${ }^{1} \mathrm{H} \mathrm{NMR}\left(400 \mathrm{MHz}, \mathrm{CDCl}_{3}\right) \delta 7.83(2 \mathrm{H}, \mathrm{d}, J=8.7 \mathrm{~Hz}, \mathrm{Ar}-\mathrm{H}), 7.38(2 \mathrm{H}, \mathrm{d}$, $J=8.7 \mathrm{~Hz}, \mathrm{Ar}-\mathrm{H}), 7.26-7.15(5 \mathrm{H}, \mathrm{m}, \mathrm{Ar}-\mathrm{H}), 4.24-4.11\left(3 \mathrm{H}, \mathrm{m}, \mathrm{COCH}_{2} \mathrm{CH}\right.$ and $\left.\mathrm{CO}_{2} \mathrm{CH}_{2}\right), 3.95(2 \mathrm{H}$, q, $\left.J=7.1 \mathrm{~Hz}, \mathrm{CO}_{2} \mathrm{CH}_{2}\right), 3.81\left(1 \mathrm{H}, \mathrm{d}, J=9.5 \mathrm{~Hz}, \mathrm{CHCO}_{2}\right), 3.52\left(1 \mathrm{H}, \mathrm{dd}, J=16.6,4.4 \mathrm{~Hz}, \mathrm{COCH}_{2}\right)$, $3.40\left(1 \mathrm{H}, \mathrm{dd}, J=16.6,9.5 \mathrm{~Hz}, \mathrm{COCH}_{2}\right), 1.24\left(3 \mathrm{H}, \mathrm{t}, J=7.1 \mathrm{~Hz}, \mathrm{CH}_{3}\right), 1.00\left(3 \mathrm{H}, \mathrm{t}, J=7.1 \mathrm{~Hz}, \mathrm{CH}_{3}\right)$; ${ }^{13} \mathrm{C}$ NMR $\left(100 \mathrm{MHz}, \mathrm{CDCl}_{3}\right) \delta 196.2,168.1,167.4,140.0,139.3,135.0,129.4,128.7,128.3,128.0$, 127.1, 61.7, 61.4, 57.5, 42.6, 40.9, 14.1, 13.8; IR (neat) 1728, 1686, 1589, 1495, 1454, 1400, 1368, 1254, 1225, 1175, 1152, 1092, 1013, 860, 829, 764, $700 \mathrm{~cm}^{-1}$; HRMS (ESI) Calcd for $\mathrm{C}_{22} \mathrm{H}_{23} \mathrm{ClNaO}_{5}$ $\left([\mathrm{M}+\mathrm{Na}]^{+}\right)$: 425.1126, Found: 425.1127; HPLC analysis: DAICEL Chiralpak AD-H, hexane/ethanol $=20: 1$, flow rate $=1.0 \mathrm{~mL} / \mathrm{min}$, retention time; $47.1 \mathrm{~min}$ (minor) and $51.5 \mathrm{~min}$ (major).

2-(3-Oxo-3-phenyl-1-pyridin-2-ylpropyl)malonic Acid Diethyl Ester (4g): $[\alpha]_{\mathrm{D}}{ }^{21}-16.2{ }^{\circ}[c$ 2.13, $\mathrm{CHCl}_{3}(90 \%$ ee $\left.)\right] ;{ }^{1} \mathrm{H} \mathrm{NMR}\left(400 \mathrm{MHz}, \mathrm{CDCl}_{3}\right) \delta 8.45(1 \mathrm{H}, \mathrm{d}, J=4.7 \mathrm{~Hz}, \mathrm{Ar}-\mathrm{H}), 7.90(2 \mathrm{H}, \mathrm{d}, J$ $=7.9 \mathrm{~Hz}, \mathrm{Ar}-\mathrm{H}), 7.54(1 \mathrm{H}, \mathrm{t}, J=7.9 \mathrm{~Hz}, \mathrm{Ar}-\mathrm{H}), 7.49(1 \mathrm{H}, \mathrm{d}, J=7.5 \mathrm{~Hz}, \mathrm{Ar}-\mathrm{H}), 7.40(1 \mathrm{H}, \mathrm{t}, J=7.5$ $\mathrm{Hz}, \mathrm{Ar}-\mathrm{H}), 7.38(2 \mathrm{H}, \mathrm{t}, J=7.9 \mathrm{~Hz}, \mathrm{Ar}-\mathrm{H}), 7.05(1 \mathrm{H}, \mathrm{dd}, J=7.5 \mathrm{~Hz}, \mathrm{Ar}-\mathrm{H}), 4.32(1 \mathrm{H}, \mathrm{dd}, J=9.9,3.6$ $\left.\mathrm{Hz}, \mathrm{CHCO}_{2}\right), 4.24-4.18\left(2 \mathrm{H}, \mathrm{m}, \mathrm{CO}_{2} \mathrm{CH}_{2}\right), 4.08\left(1 \mathrm{H}, \mathrm{d}, J=9.9 \mathrm{~Hz}, \mathrm{COCH}_{2} \mathrm{CH}\right), 4.03-3.95(2 \mathrm{H}, \mathrm{m}$, $\left.\mathrm{CO}_{2} \mathrm{CH}_{2}\right), 3.80\left(1 \mathrm{H}, \mathrm{dd}, J=17.4,9.9 \mathrm{~Hz}, \mathrm{COCH}_{2}\right), 3.42\left(1 \mathrm{H}, \mathrm{dd}, J=17.4,3.6 \mathrm{~Hz}, \mathrm{COCH}_{2}\right), 1.24$ $\left(3 \mathrm{H}, \mathrm{t}, J=7.1 \mathrm{~Hz}, \mathrm{CH}_{3}\right), 1.05\left(3 \mathrm{H}, \mathrm{t}, J=7.1 \mathrm{~Hz}, \mathrm{CH}_{3}\right) ;{ }^{13} \mathrm{C} \mathrm{NMR}\left(100 \mathrm{MHz}, \mathrm{CDCl}_{3}\right) \delta 197.4,168.3$, $167.7,160.1,148.7,136.5,135.9,132.8,128.3,127.8,124.7,121.6,61.5,61.2,41.7,41.5,14.0$, 13.8; IR (neat) 1728, 1684, 1591, 1474, 1449, 1437, 1368, 1300, 1256, 1227, 1209, 1152, 1096, 1030, 1001, 860, 787, 750, $691 \mathrm{~cm}^{-1}$; HRMS (ESI) Calcd for $\mathrm{C}_{21} \mathrm{H}_{24} \mathrm{NO}_{5}\left([\mathrm{M}+\mathrm{H}]^{+}\right): 370.1649$, Found: 370.1649. HPLC analysis: DAICEL Chiralpak OJ-H, hexane/ethanol $=9: 1$, flow rate $=1.0 \mathrm{~mL} / \mathrm{min}$, retention time; $12.6 \mathrm{~min}$ (minor) and $14.0 \mathrm{~min}$ (major). 
2-(1-Furan-2-yl-3-oxo-3-phenylpropyl)malonic Acid Diethyl Ester (4h): $[\alpha]_{\mathrm{D}}^{29}-1.34{ }^{\circ}[c 0.37$, $\mathrm{CHCl}_{3}$ (86\% ee)]; ${ }^{1} \mathrm{H} \mathrm{NMR}\left(400 \mathrm{MHz}, \mathrm{CDCl}_{3}\right) \delta 7.95(2 \mathrm{H}, \mathrm{d}, J=8.0 \mathrm{~Hz}, \mathrm{Ar}-\mathrm{H}), 7.55(1 \mathrm{H}, \mathrm{t}, J=8.0$ $\mathrm{Hz}, \operatorname{Ar}-\mathrm{H}), 7.43(2 \mathrm{H}, \mathrm{t}, J=8.0 \mathrm{~Hz}, \mathrm{Ar}-\mathrm{H}), 7.26(2 \mathrm{H}, \mathrm{s}, \mathrm{Ar}-\mathrm{H}), 6.21(1 \mathrm{H}, \mathrm{m}, \mathrm{Ar}-\mathrm{H}), 6.11(2 \mathrm{H}, \mathrm{d}, J=$ $3.6 \mathrm{~Hz}, \mathrm{Ar}-\mathrm{H}), 4.33\left(1 \mathrm{H}, \mathrm{ddd}, J=8.8,8.0,4.8 \mathrm{~Hz}, \mathrm{COCH}_{2} \mathrm{C} \underline{\mathrm{H}}\right), 4.23-4.15\left(2 \mathrm{H}, \mathrm{m}, \mathrm{CO}_{2} \mathrm{CH}_{2}\right), 4.10$ $\left(2 \mathrm{H}, \mathrm{q}, J=7.2 \mathrm{~Hz}, \mathrm{CO}_{2} \mathrm{CH}_{2}\right), 3.90\left(2 \mathrm{H}, \mathrm{d}, J=8.0 \mathrm{~Hz}, \mathrm{CHCO}_{2}\right), 3.58(1 \mathrm{H}, \mathrm{dd}, J=17.2,8.8 \mathrm{~Hz}$, $\left.\mathrm{COCH}_{2}\right), 3.47\left(1 \mathrm{H}, \mathrm{dd}, J=17.2,4.8 \mathrm{~Hz}, \mathrm{COCH}_{2}\right), 1.24\left(3 \mathrm{H}, \mathrm{t}, J=7.2 \mathrm{~Hz}, \mathrm{CH}_{3}\right), 1.16(3 \mathrm{H}, \mathrm{t}, J=7.2$ $\left.\mathrm{Hz}, \mathrm{CH}_{3}\right) ;{ }^{13} \mathrm{C} \mathrm{NMR}\left(100 \mathrm{MHz}, \mathrm{CDCl}_{3}\right) \delta 197.0,167.8,167.6,153.4,141.4,136.6,133.0,128.5$, 128.0, 110.1, 107.0, 61.6, 61.5, 55.2, 39.9, 34.3, 14.1, 14.0; IR (neat) 1730, 1686, 1597, 1449, 1368, 1255, 1223, 1153, 1096, 1013, 860, 737, $690 \mathrm{~cm}^{-1}$; HRMS (ESI) Calcd for $\mathrm{C}_{20} \mathrm{H}_{22} \mathrm{NaO}_{6}\left([\mathrm{M}+\mathrm{Na}]^{+}\right)$: 381.1309, Found: 381.1305; HPLC analysis: DAICEL Chiralpak AD-H, hexane/ethanol = 20:1, flow rate $=0.5 \mathrm{~mL} / \mathrm{min}$, retention time; $49.9 \mathrm{~min}$ (major) and $54.1 \mathrm{~min}$ (minor).

2-(3-Oxo-3-phenyl-1-thiophen-2-ylpropyl)malonic Acid Diethyl Ester (4i): $[\alpha]_{\mathrm{D}}{ }^{20}-13.5^{\circ}[c$ 1.66, $\mathrm{CHCl}_{3}\left(94 \%\right.$ ee)]; ${ }^{1} \mathrm{H} \mathrm{NMR}\left(400 \mathrm{MHz}, \mathrm{CDCl}_{3}\right) \delta 7.72(1 \mathrm{H}, \mathrm{d}, J=4.7 \mathrm{~Hz}, \mathrm{Ar}-\mathrm{H}), 7.57(1 \mathrm{H}, \mathrm{d}, J$ $=4.7 \mathrm{~Hz}, \mathrm{Ar}-\mathrm{H}), 7.28-7.21(4 \mathrm{H}, \mathrm{m}, \mathrm{Ar}-\mathrm{H}), 7.16(1 \mathrm{H}, \mathrm{t}, J=6.7 \mathrm{~Hz}, \mathrm{Ar}-\mathrm{H}), 7.07(1 \mathrm{H}, \mathrm{dd}, J=8.7,4.7$ $\mathrm{Hz}, \mathrm{Ar}-\mathrm{H}), 4.24-4.13\left(3 \mathrm{H}, \mathrm{m}, \mathrm{COCH}_{2} \mathrm{CH}\right.$ and $\left.\mathrm{CO}_{2} \mathrm{CH}_{2}\right), 3.94\left(2 \mathrm{H}, \mathrm{q}, J=7.1 \mathrm{~Hz}, \mathrm{CO}_{2} \mathrm{CH}_{2}\right), 3.83(1 \mathrm{H}$, $\left.\mathrm{d}, J=9.5 \mathrm{~Hz}, \mathrm{CHCO}_{2}\right), 3.46\left(1 \mathrm{H}, \mathrm{dd}, J=16.2,4.7 \mathrm{~Hz}, \mathrm{COCH}_{2}\right), 3.37(1 \mathrm{H}, \mathrm{dd}, J=16.2,9.5 \mathrm{~Hz}$, $\left.\mathrm{COCH}_{2}\right), 1.24\left(3 \mathrm{H}, \mathrm{t}, J=7.1 \mathrm{~Hz}, \mathrm{CH}_{3}\right), 0.99\left(3 \mathrm{H}, \mathrm{t}, J=7.1 \mathrm{~Hz}, \mathrm{CH}_{3}\right) ;{ }^{13} \mathrm{C} \mathrm{NMR}\left(100 \mathrm{MHz}, \mathrm{CDCl}_{3}\right)$ ठ 190.1, 168.0, 167.4, 143.9, 139.9, 133.4, 131.9, 128.2, 128.0, 127.9, 127.0, 61.6, 61.3, 57.4, 43.3, 41.2, 14.0, 13.8; IR (neat) 1728, 1661, 1518, 1497, 1454, 1414, 1368, 1256, 1233, 1152, 1096, 1028, 912, 858, 727, $700 \mathrm{~cm}^{-1}$; HRMS (ESI) Calcd for $\mathrm{C}_{20} \mathrm{H}_{22} \mathrm{NaO}_{5} \mathrm{~S}\left([\mathrm{M}+\mathrm{Na}]^{+}\right)$: 397.1080, Found: 397.1078; HPLC analysis: DAICEL Chiralpak AD-H, hexane/ethanol $=4: 1$, flow rate $=1.0 \mathrm{~mL} / \mathrm{min}$, retention time; $19.9 \mathrm{~min}$ (minor) and $22.3 \mathrm{~min}$ (major).

2-(3-Oxo-1-phenyl-3-thiophen-2-ylpropyl)malonic Acid Diethyl Ester (4j): $[\alpha]_{\mathrm{D}}{ }^{18}-14.3^{\circ}[c$ 1.39, $\mathrm{CHCl}_{3}(94 \%$ ee $\left.)\right] ;{ }^{1} \mathrm{H} \mathrm{NMR}\left(400 \mathrm{MHz}, \mathrm{CDCl}_{3}\right) \delta 7.72(1 \mathrm{H}, \mathrm{d}, J=3.6 \mathrm{~Hz}, \mathrm{Ar}-\mathrm{H}), 7.57(1 \mathrm{H}, \mathrm{d}, J$ $=5.1 \mathrm{~Hz}, \mathrm{Ar}-\mathrm{H}), 7.28-7.21(4 \mathrm{H}, \mathrm{m}, \mathrm{Ar}-\mathrm{H}), 7.16(1 \mathrm{H}, \mathrm{t}, J=6.7 \mathrm{~Hz}, \mathrm{Ar}-\mathrm{H}), 7.07(1 \mathrm{H}, \mathrm{dd}, J=5.1,3.6$ $\mathrm{Hz}, \mathrm{Ar}-\mathrm{H}), 4.24-4.13\left(3 \mathrm{H}, \mathrm{m}, \mathrm{COCH}_{2} \mathrm{CH}\right.$ and $\left.\mathrm{CO}_{2} \mathrm{CH}_{2}\right), 3.94\left(2 \mathrm{H}, \mathrm{q}, J=7.1 \mathrm{~Hz}, \mathrm{CO}_{2} \mathrm{CH}_{2}\right), 3.83(1 \mathrm{H}$, $\left.\mathrm{d}, J=9.4 \mathrm{~Hz}, \mathrm{CHCO}_{2}\right), 3.46\left(1 \mathrm{H}, \mathrm{dd}, J=16.2,4.7 \mathrm{~Hz}, \mathrm{COCH}_{2}\right), 3.37(1 \mathrm{H}, \mathrm{dd}, J=16.2,9.4 \mathrm{~Hz}$, $\left.\mathrm{COCH}_{2}\right), 1.24\left(3 \mathrm{H}, \mathrm{t}, J=7.1 \mathrm{~Hz}, \mathrm{CH}_{3}\right), 1.00\left(3 \mathrm{H}, \mathrm{t}, J=7.1 \mathrm{~Hz}, \mathrm{CH}_{3}\right) ;{ }^{13} \mathrm{C} \mathrm{NMR}\left(100 \mathrm{MHz}, \mathrm{CDCl}_{3}\right)$ ठ 190.1, 168.0, 167.4, 143.9, 139.9, 133.5, 131.9, 128.2, 128.0, 127.9, 127.0, 61.6, 61.3, 57.4, 43.3, 41.2, 14.1, 13.8; IR (neat) 1728, 1661, 1518, 1454, 1414, 1368, 1256, 1234, 1152, 1096, 1028, 912 , 858, 727, $700 \mathrm{~cm}^{-1}$; HRMS (ESI) Calcd for $\mathrm{C}_{20} \mathrm{H}_{22} \mathrm{NaO}_{5} \mathrm{~S}\left([\mathrm{M}+\mathrm{Na}]^{+}\right)$: 397.1080, Found: 397.1065 ; HPLC analysis: DAICEL Chiralpak AD-H, hexane/ethanol $=4: 1$, flow rate $=1.0 \mathrm{~mL} / \mathrm{min}$, retention time; $19.5 \mathrm{~min}$ (minor) and $21.9 \mathrm{~min}$ (major).

\section{Reference}

(1) Ooi, T.; Ohara, D.; Tamura, M.; Maruoka, K. J. Am. Chem. Soc. 2004, 126, 6844. 